\title{
Integración de la prevención en el diseño de obras de construcción: relación con la siniestralidad laboral, análisis de su regulación normativa, bases conceptuales y desarrollo internacional
}

\author{
Prevention through design: relation to construction \\ fatalities, analysis of legal requirements, conceptual \\ foundations and international development
}

C. Arévalo ${ }^{(*)}$

RESUMEN

La gestión de la prevención en las obras de construcción en España presenta evidentes signos de estancamiento que parecen estar más relacionados con el formalismo y la ausencia de innovación de los que adolecen, con carácter general, las actuaciones de los agentes implicados en la misma que con la crisis económica y de actividad que sufre el sector. Ante esta situación, el presente artículo analiza la influencia que las decisiones tomadas en el proyecto guardan con la siniestralidad laboral de las obras y las diferencias entre la regulación de la gestión preventiva a llevar a cabo en dicha fase en los ámbitos comunitario y español. Así mismo, se recopilan diversas iniciativas internacionales en relación con el concepto de "Prevención a través del Diseño" (Ptd) basadas en la anticipación de la gestión de los aspectos preventivos a las etapas más tempranas del proceso.

$750-20$

Palabras clave: Integración de la prevención; proyecto; construcción; siniestralidad laboral; prevención a través del diseño; PtD.

\section{SUMMARY}

Health \& Safety management in construction in Spain shows signs of stagnation appear to be related more to the formalism and lack of innovation from which they suffer the actions of the agents involved in it than to the economic crisis that's facing the sector activity. Against this background, this article attempts to analyze the role that architectural and planning and organizational options taken in the Project preparation stage play in occupational accidents occurring on construction sites. Secondly, it aims to identify the differences between the regulation of Health \& Safe management to carry out at this stage at the EU and Spain. Finally, the article collects relevant researches in relation to the concept of "Prevention through Design" (Ptd) and "Design for Construction Safety" as the process in which designers explicitly consider safety issues during the design process in order to influence in the safety of the whole project life cycle.

Keywords: Prevention through design; design for safety; construction site accidents; construction safety; occupational safety.

(*) ETSICCP-Universidad Politécnica de Madrid (España). 


\section{ANÁLISIS DE LA RELACIÓN EXISTENTE ENTRE LAS DECISIONES TÉCNICAS ADOPTADAS EN LA FASE PROYECTO $Y$ LA SINIESTRALIDAD LABORAL EN LAS OBRAS DE CONSTRUCCIÓN}

A pesar del vertiginoso descenso de actividad sufrido por el sector de la construcción en España en los últimos cuatro años, éste sigue concentrando buena parte de los accidentes graves y mortales acaecidos en nuestro país. De esta manera, a los 1.180 accidentes graves y 134 mortales registrados en el sector en 2010 se debe añadir que sus índices de siniestralidad, en cualquiera de sus modalidades oficiales, siguen duplicando con amplitud los registros medios del global de los sectores productivos ${ }^{1}$.

Si bien los expertos aceptan que las condiciones intrínsecas del sector $-y$ hasta su propio ordenamiento jurídico en la materia (1)- dificultan claramente una gestión preventiva eficaz, no es menos cierto que dichas particularidades son ya conocidas por los agentes intervinientes en el sector y que, en absoluto, resultan novedosas o insalvables. De esta manera, y sin por ello obviar que la siniestralidad laboral se configura como un fenómeno multicausal, resulta necesario ahondar en el estudio de aquellos factores técnicos que guardan una relación más directa con el problema específico de la accidentalidad en el sector de la construcción.

En dicho ámbito, no resulta novedosa la correlación establecida hace ya más de dos décadas por el conocido informe Lorent (2) que, a la hora de determinar el origen de las causas de los accidentes mortales registrados en el sector, determina que:

- El 35\% del total de accidentes mortales registrados en obras de construcción se deben a decisiones tomadas en la fase de diseño de la obra.

- El 28\% a deficiencias en la organización y planificación de los trabajos que se lleva a cabo con carácter previo al comienzo de la obra.

- El 37\% restante tiene su origen en cuestiones relacionadas con la fase de ejecución.

${ }^{1}$ Datos de la Estadística de accidentes de trabajo y enfermedades profesionales del Ministerio de Empleo y Seguridad Social de 2010

${ }^{2}$ Buena muestra de ello es su inclusión en la exposición de motivos de la Directiva 92/57/CEE del Consejo, de 24 de junio de 1992, relativa a las disposiciones mínimas de seguridad y de salud que deben aplicarse en las obras de construcción temporales o móviles (en adelante Directiva 92/57/CEE).
Sin entrar a cuestionar dichas conclusiones ni, en particular, la eventual vinculación entre los presuntos defectos en la organización y planificación de los trabajos y las decisiones tomadas en la fase de proyecto, lo cierto es que el citado informe sentó las bases para aceptar de manera generalizada que las actuaciones desarrolladas en fase de proyecto guardan una relación cierta con la seguridad de los trabajos de ejecución de las obras ${ }^{2}$.
Superado el citado informe, se debe constatar que la posible vinculación entre el diseño de la obra efectuado en el proyecto y la siniestralidad laboral registrada en la fase de ejecución ha motivado numerosos estudios de investigación a nivel internacional entre los que se destacan, por su rigurosidad, los siguientes:

1. En el Reino Unido, el HSE (3) analizó 100 accidentes acaecidos en obras de construcción en el Reino Unido deduciendo que en prácticamente el 50\% de los mismos se podrían haber disminuido los riesgos que los ocasionaron mediante la adopción de soluciones en fase de diseño. Con anterioridad, Smallwood (4) ya había adscrito a las deficiencias en el diseño el $50 \%$ de los problemas de seguridad y salud laboral de la construcción.

2. También en el Reino Unido, Bennet (5) analizó 71 accidentes del sector de la construcción relacionando más del $60 \%$ de los mismos con mejoras a adoptar en la fase de diseño atreviéndose a concluir que en 39 de ellos se apreciaba responsabilidad directa por insuficiencias en el proyecto de la obra.

3. En la misma línea, Haslam (6) postula la preponderancia causal de los factores definidos en las fases previas a la ejecución de las obras (exigencias del cliente, limitaciones económicas y formación de los agentes) sobre los factores relacionados con la fase de ejecución (actitud de lo trabajadores, condiciones del lugar de trabajo y condiciones de los materiales y equipos de la obra).

4. En EE.UU., constan sendos informes del Centro de Protección de Derechos de los Trabajadores, CPWR (7) (8), en los que se analizaron un total de 450 informes de accidentes graves y mortales acaecidos en el sector de la construcción. A este respecto, los informes concluyeron que casi un tercio de los mismos (136) estuvieron vinculados o se podrían haber evitado mediante la adopción de medidas en el diseño de la obra.

5. Por último, la Comisión Nacional de Seguridad y Salud Ocupacional del Gobierno de Australia (9) admite que más del $30 \%$ de los 2.705 accidentes analizados en obras de construcción en Australia guardaron relación con el diseño de la obra.

En base a dichos estudios internacionales, se puede afirmar que las decisiones de diseño tomadas en la fase de proyecto guardan una relación cierta con la siniestralidad laboral y las condiciones de trabajo existentes en la fase de ejecución (variando dicha influencia, según el autor, entre el 30 y el $50 \%$ de los accidentes analizados). 
En lo que se refiere al sector de la construcción en España, se debe constatar que las estadísticas e informes de siniestralidad consultados, aún mereciendo la pena citar por su extensión y rigor los desarrollados por SEOPAN (10) y la Fundación MUSAAT (11), no resultan representativos a este respecto pues se limitan a analizar los factores causales relacionados con cada accidente sin entrar a valorar la eventual contribución de las decisiones técnicas tomadas en la fase de proyecto.

\section{REGULACIÓN NORMATIVA DE LA INTEGRACIÓN DE LA PREVENCIÓN EN EL PROYECTO DE CONSTRUC- CIÓN: COMPARACIÓN ENTRE EL ORDENAMIENTO A NIVEL COMUNI- TARIO Y ESPAÑOL}

La integración de la prevención en la fase de diseño fue uno de los principios inspiradores de la Directiva 92/57/CEE. Dicha norma, dictada con carácter específico para ordenar la gestión de la seguridad y salud en las obras de construcción, no sólo reconoció la vinculación entre los accidentes de trabajo y las decisiones y planificación llevadas a cabo en esta fase sino que, además, incluyó en su exposición de motivos la necesidad de "reforzar la coordinación entre las distintas partes que intervienen ya desde la fase de proyecto...".

Con el objetivo de lograr la perseguida integración de la prevención en esta fase, el legislador comunitario dedicó dos de los catorce artículos de la Directiva a las actuaciones a desarrollar en el proyecto. Dichos artículos, el 4 y el 5, recogen tanto los principios a considerar en la elaboración de proyecto como las actuaciones a desarroIlar por los coordinadores de seguridad en esta fase ${ }^{3}$.

En relación con los principios preventivos a considerar en esta fase por los proyectistas y la propiedad de la obra ${ }^{4}$, se debe destacar la referencia expresa a la obligada aplicación de los principios generales de prevención a las decisiones técnicas, organizativas y de planificación a tomar por los citados agentes en la etapa de proyecto (Figura 1).
De esta manera, la pretensión del legislador comunitario no es otra que lograr que la organización, la planificación y el diseño de la actividad productiva de la obra que, por definición efectúa el proyectista en esta etapa, se vea influenciada por prioridades preventivas. En base a todo ello, se entiende que decisiones y aspectos tales como los plazos previstos para cada fase de la obra, la propio programación/plan de obra o las características materiales, geométricas y constructivas de los diferentes elementos diseñados deben considerar los principios previamente enunciados.

En lo que se refiere a la actuación de los coordinadores en materia de seguridad y salud $^{5}$ en fase de proyecto (Art. 6), la Directiva 92/57/CEE les traslada tanto la coordinación de la aplicación de los principios preventivos aludidos, como el establecimiento de un plan de seguridad y salud, definido como el conjunto de normas y medidas aplicables a la obra, y la constitución de un expediente informativo para los trabajos a efectuar en fase de servicio (expediente adaptado).

De esta manera, la Directiva no sólo localiza en la fase de proyecto el origen de buena parte de los riesgos laborales de la futura ejecución y explotación de la obra sino que, con carácter dispositivo, regula las actuaciones a llevar a cabo en materia preventiva por los proyectistas, la propiedad y los coordinadores en materia de seguridad y salud en fase de proyecto (en adelante, CSS/P), con la finalidad clara de procurar la integración de la prevención en las etapas más tempranas del proceso y evitar que, en la práctica, los aspectos preventivos se configuren como un elemento añadido a posteriori a la definición técnica de la obra.

En cuanto a la trasposición de la Directiva 92/57/CEE a los diferentes estados miembros, se coincide con Martínez Aires et al (12) en que el resultado y la efectividad de tales trasposiciones ha resultado manifiestamente desigual. En lo que se refiere a su desarrollo en España, el proceso de trasposición se vio fuertemente influenciado por

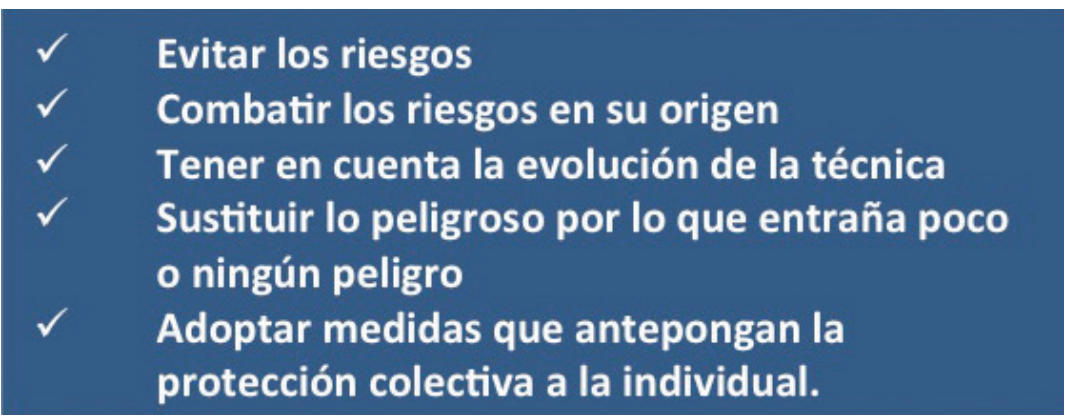

1. Resumen de los principales principios preventivos a aplica en el proceso de diseño y elaboración del proyecto.

${ }^{3}$ Debe constatarse que, a diferencia de lo que ocurre con el RD 1627/97, la Directiva 92/57/CEE no vincula la presencia del Coordinador en fase de proyecto a la concurrencia de más de un proyectista sino a la concurrencia de más de un empresario (contratista, subcontratista o trabajador autónomo) en la fase de ejecución.

${ }^{4}$ Término que en la transposición española, sin una justificación clara ni razonada, se convirtió en el promotor de la obra.

${ }^{5}$ Tal y como se analiza más adelante, las referencias al plan de seguridad y salud en fase de proyecto de la Directiva 92/57/CEE se trasladaron, en nuestro país, al Estudio de Seguridad y Salud. 
2. Diferencias entre la regulación de la gestión de la prevención en la fase de proyecto en la Directiva Comunitaria 92/57/ CEE y su trasposición al ordenamiento jurídico español, el RD $1627 / 97$ el preexistente RD 555/86, optándose por un modelo híbrido entre ambos referentes normativos. Esta técnica legislativa condujo a que el texto reglamentario resultante, el vigente RD 1627/97, presente evidentes notas discordantes en lo que se refiere al modelo de gestión preventiva que la Directiva 92/57/ CEE establecía para la fase de proyecto. En esencia, las discordancias (Figura 2) se concentran en los siguientes aspectos:

- En primer lugar, mientras que la Directiva Comunitaria responsabiliza a la propiedad de la obra de la integración de la prevención en la fase de proyecto (Art.4), el RD 1627/97 se limita a vincular tal integración a la actuación del proyectista (Art. 8).

- En cuanto a la figura del CSS/P, el desarrollo reglamentario español vincula la exigencia de su designación a la participación en la elaboración del proyecto de más de un proyectista (Art. 3.1). Sin entrar a valorar si los múltiples técnicos que suelen, en la práctica, intervenir en la elaboración de un proyecto tienen la condición de "proyectista" a efectos de la aplicación de tal precepto, resulta incuestionable que esta limitación modifica la regulación que el legislador comunitario llevó a cabo de la gestión de la prevención en la fase de proyecto.

- Más evidente resulta la dualidad documental introducida en la trasposición española que, en vez de ordenar la gestión de la prevención alrededor de un único documento (el Plan de Seguridad y Salud que se elabora con el proyecto y se adapta en fase de ejecución), optó por crear la dupla Estudio/Plan de Seguridad y Salud a elaborar por diferentes agentes en las fase de proyecto y ejecución.

- Por último, el RD 1627/97 optó por eliminar el expediente adaptado y trasladar la información preventiva de interés para los posteriores trabajos de mantenimiento de la obra al Estudio o Estudio Básico de Seguridad y Salud. La principal diferencia a este respecto radica en que mientras el expediente adaptado se configura en la

\begin{tabular}{|l|l|l|}
\cline { 2 - 3 } & \multicolumn{2}{|c|}{} \\
\cline { 2 - 3 } & $\begin{array}{l}\text { Regulación Comunitaria } \\
\text { (Directiva 92/57/CEE) }\end{array}$ & $\begin{array}{l}\text { Trasposición Española } \\
\text { (RD 1627/97) }\end{array}$ \\
\hline $\begin{array}{l}\text { Deber de integrar los principios } \\
\text { preventivos en la elaboración } \\
\text { del proyecto }\end{array}$ & $\begin{array}{l}\text { Propiedad y director de } \\
\text { obra (equivalente a } \\
\text { proyectista) }\end{array}$ & Proyectista \\
\hline $\begin{array}{l}\text { Obligación de designar } \\
\text { Coordinador en fase de } \\
\text { proyecto }\end{array}$ & $\begin{array}{l}\text { Todas las obras más de } \\
\text { una empresa }\end{array}$ & Más de un proyectista \\
\hline $\begin{array}{l}\text { Herramientas documentales en } \\
\text { fase de proyecto }\end{array}$ & $\begin{array}{l}\text { Plan de Seguridad y Salud } \\
\text { y expediente adaptado }\end{array}$ & $\begin{array}{l}\text { Estudio de Seguridad y Salud } \\
\text { (Plan en fase de ejecución) }\end{array}$ \\
\hline $\begin{array}{l}\text { Información para los trabajos } \\
\text { posteriores }\end{array}$ & Expediente adaptado & $\begin{array}{l}\text { Incluido en el Estudio de } \\
\text { Seguridad }\end{array}$ \\
\hline
\end{tabular}

legislación comunitaria como un documento vivo que se actualiza a lo largo de la construcción de la obra, en España, el Estudio de Seguridad y Salud se vincula al proyecto lo que, en la práctica, acaba por limitar su eventual actualización y adaptación en fase de ejecución.

Si bien las diferencias reguladoras de la gestión preventiva en la fase de proyecto parecen claras, más parejas resultan las valoraciones que, en los ámbitos comunitario y español, se realizan de dicha integración. A este respecto, la Comisión Europea publicó en 2008 (13) un estudio específico en relación con el nivel de integración de la prevención en los proyectos de construcción elaborados en los diferentes estados miembros concluyendo que:

- En la fase de elaboración del proyecto existe generalmente una "falta de coordinación y de control" limitándose, en general, la coordinación a la fase de construcción.

- El principal aspecto a mejorar en los estados miembros son "las carencias en el desarrollo de la coordinación de la seguridad en la fase de preparación". A este mismo respecto, se llega a señalar categóricamente que: "si no se ha designado el coordinador antes de la fase de ejecución del proyecto, se ha incumplido la obligación de incorporar los principios de la prevención en la preparación del proyecto".

- En el apartado de valoración de la actuación de los proyectistas, ingenieros y arquitectos se concluye que ésta resulta insuficiente pues no incluye la prevención como "un factor principal a la hora de tomar decisiones arquitectónicas o de concepción".

Dos años más tarde, la propia Comisión Europea desarrolló una serie de recomendaciones no vinculantes para la aplicación de la Directiva 92/57/CEE (14) incluyendo, entre éstas, un apartado específico para promover la integración de la prevención en la fase proyecto. Dichas recomendaciones identifican a los proyectistas como agentes críticos para la mejora preventiva de las obras gracias a su capacidad para identificar y eliminar condiciones de riesgo desde las etapas más tempranas del proyecto.

De igual manera, las citadas recomendaciones incluyen la prescripción de evitar la práctica habitual de no considerar los aspectos preventivos de la futura obra hasta que se ha terminado el diseño del proyecto. Por último, y en relación con los aspectos en los que la actuación del proyectista puede resultar crítica, se recogen materias tales como la identificación de los riesgos 
del entorno, el retranqueo o descarga de líneas eléctricas, el control de los tráficos existentes, la planificación temporal de los trabajos o el plan logístico de la futura obra.

Si bien se considera que las apreciaciones realizadas por la Comisión Europea en ambos documentos resultan claramente aplicables a la situación del sector de la construcción en España, cabe indicar que la identificación de la fase de proyecto como etapa crítica para mejorar la gestión preventiva de las obras de construcción en nuestro país también se recoge por expertos en la materia (15). En base a todo ello, se debe concluir que las etapas de planeamiento y elaboración del proyecto presentan, también en nuestro país, un potencial preventivo desaprovechado en la actualidad.

\section{PREVENCIÓN A TRAVÉS DEL DISEÑO (PTD): ANTECEDENTES, ANÁLISIS CONCEPTUAL Y DESARROLLO INTERNACIONAL}

\subsection{Antecedentes}

La integración de la prevención a través del diseño de la obras de construcción es un concepto que se ha ido desarrollando en los últimos 15 años en ciertos países de nuestro entorno. Llama la atención que, al menos en lo que refiere a su desarrollo en el ámbito norteamericano, tal avance no ha derivado de la aplicación de disposición normativa alguna que regulen tal actuación sino que, más bien, ha surgido de la propia utilidad y potencial que puede llegar a suponer la adopción de dichas soluciones.

El análisis cronológico de los orígenes de la $\mathrm{PtD}^{6}$ cubre los siguientes hitos:

- En 1985, la Oficina Internacional del Trabajo (16) identificó la necesidad de que los proyectistas estuvieran involucrados y considerarse en su trabajo los aspectos relacionados con la seguridad y salud en la construcción.

- El 23 de abril de 1987 fallecieron 28 trabajadores en el accidente en la construcción torre L’Ambiance Plaza (17) A raíz del mismo, se tramitaron sendas propuestas ante el Congreso de los EEUU para incrementar la responsabilidad preventiva de los proyectistas que fueron desestimadas en base a la oposición de parte del sector.

- La Fundación Europea para la mejora de las condiciones de vida y trabajo concluyó en 1991 que el 60\% de los accidentes mortales en construcción guardaban relación con decisiones llevadas acabo en etapas previas al comienzo de las obras.

- En EE.UU, en 1992 F. Wiegand y J. Hinze (18) concluyeron que la seguridad de los

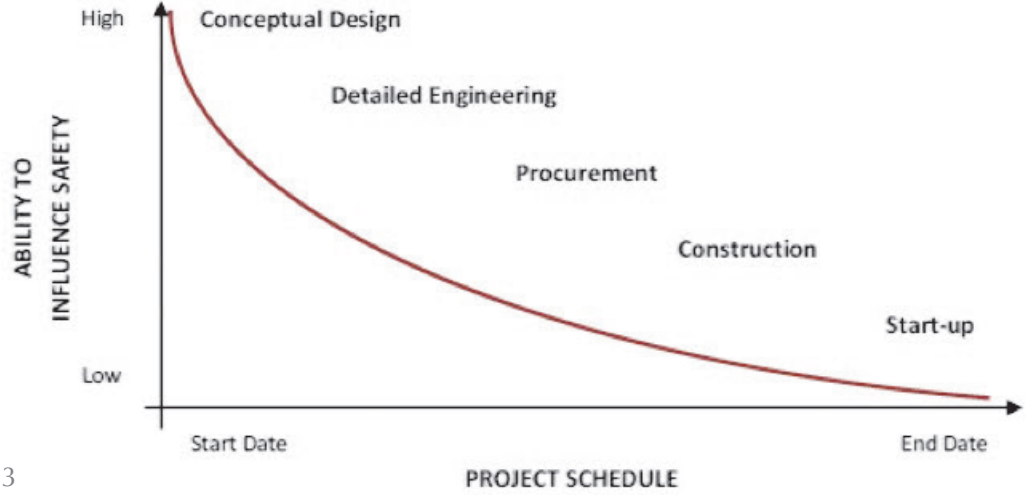

trabajadores de la construcción no puede estar garantizada únicamente por la legislación, recalcando el papel fundamental de los diseñadores en la prevención de accidentes. En la misma línea, J. Gambatese (19) desarrolló en 1997 un conjunto de prácticas para promover el diseño para la seguridad en la construcción.

- Igualmente, y en lo que se refiere a EE.UU., Szymberski (20) concluyó en su análisis de la industria de la construcción que la mejora de la prevención pasa por que la seguridad sea una de las consideraciones primordiales en las fases de diseño conceptual y preliminar. Su curva de influencia tiempo/seguridad, pone de manifiesto que una parte significante de la capacidad de mejorar las condiciones de trabajo de la obra se pierde cuando dichas consideraciones se retrasan hasta la fase de construcción. De esta manera, retrasar las decisiones y actuaciones preventivas a la fase de ejecución no sólo resta eficacia a las mismas sino que supone desaprovechar las ventajas que ofrece la planificación en la materia y, en definitiva, el diseñar de manera eficaz para la eliminación, prevención y reducción de los riesgos laborales (Figura 3).

- La OHSA calificó en 1999 la PtD como algo "necesario" para materializar el avance en la gestión preventiva de las obras (21).

- En lo que respecta al Reino Unido, la PtD se requiere desde 1995 por la norma Construction (Design and Management) Regulations (transposición de la Directiva 92/57/CEE actualizada en 2007). De esta manera, la necesaria identificación de riesgos en el proyecto obliga al proyectista a tomar medidas y decisiones técnicas, ingenieriles y arquitectónicas encaminadas a su eliminación y control. Así, la regulación normativa de la gestión de la prevención en la construcción (CDM Regulations), dedica un artículo exclusivo, el 11, a definir los deberes preventivos del proyectista incluyendo entre ellos las siguientes obligaciones:

- Considerar en el diseño la seguridad de los trabajadores en la ejecución de la obra.
3. Curva de influencia Tiempo/ Seguridad (Szymberski, 1997).

\footnotetext{
${ }^{6}$ Las denominaciones Prevención a través del diseño (Prevention Through Design o PtD) y Diseño para la seguridad en la construcción (Designing for Construction Safety, o DfCS) corresponden al mismo concepto si bien mientras que la primera de ellas procede del ámbito británico, la segunda se origina en el norteamericano.
} 


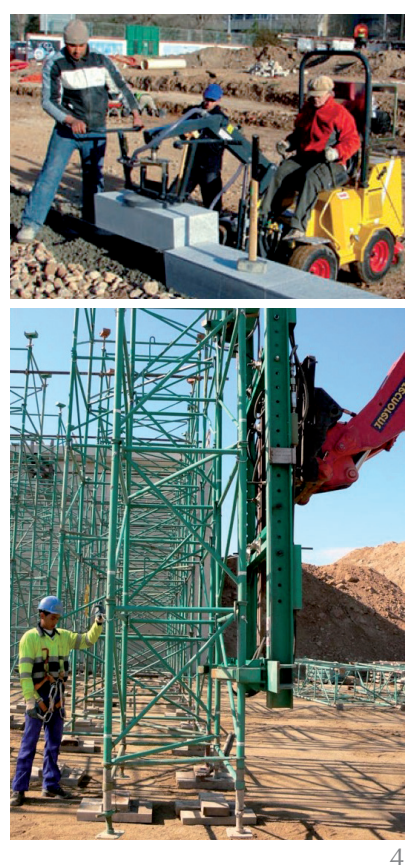

4. Soluciones técnicas para mejorar las condiciones de trabajo de actividades de montaje de cimbras cuajadas y la colocación de bordillos (25).
- Evitar los riesgos previsibles tanto en lo referente a la ejecución como al mantenimiento (limpieza de ventanas y cubiertas y mantenimiento de instalaciones) como al entorno de la obra (terceros).

- Priorizar las protecciones colectivas e informar al resto de agentes.

- Otros países comunitarios como Suecia, Dinamarca, Finlandia, Reino Unido y la República de Irlanda han ido asignado responsabilidades específicas a los proyectistas en relación con la eliminación de riesgos mediante la adopción de soluciones ingenieriles y arquitectónicas en fase de proyecto.

\subsection{Definición de la PtD y análisis conceptual de sus fundamentos.}

Las técnicas de Prevención a través del Diseño se consideran incluidas en el concepto general de integración de la prevención en la elaboración del proyecto e incluyen entre sus fundamentos conceptuales los siguientes:

- Exigen la consideración de la seguridad de los trabajos de construcción y mantenimiento y explotación de la futura obra desde la concepción de la misma.

- Persiguen mejorar las condiciones de trabajo y minimizar los riesgos en los trabajos de ejecución y mantenimiento del edificio, instalación o infraestructura proyectada.

- De acuerdo con Gambastese (22) precisan que el proyectista asuma la mejora de la seguridad de la obra a través de su actuación de forma que, en la práctica, incluya entre sus cometidos y objetivos los siguientes:

- Adaptar las características, planificación, materiales y configuración definitiva del proyecto considerando los aspectos relacionados con la seguridad y salud de los trabajos a ejecutar

- Prescribir desde el proyecto soluciones de diseño que mejoren las condiciones de trabajo de la obra.

- Informar al resto de agentes de los riesgos que no han podido ser evitados a las empresas que ejecuten la obra.

De esta manera, las técnicas de PtD acarrean la modificación de las características permanentes del proyecto de construcción con el fin de garantizar que la seguridad de los trabajos de construcción. Todo ello, supone que la citada integración debe extenderse al conjunto íntegro del proyecto incluyendo su consideración en aspectos tales como la definición técnica de soluciones constructivas, el plan de obra, las prescripciones técnicas o el presupuesto.

Adicionalmente, las técnicas de PtD incluyen el análisis de actividades y métodos de construcción desde la óptica preventiva concluyendo con la propuesta de nuevas soluciones constructivas (a veces basadas en aportaciones de sencilla implementación) que mejoran las condiciones de trabajo en las que se ejecutarán dichas actividades. Estas soluciones han sido desarrolladas tanto en relación con los procesos de prefabricación, Bendig \& Gibb (23), como para la prevención de lesiones músculo esqueléticas, Kramer (24), actuaciones en andamios suspendidos, Saurin \& Buarque de Macedo (25), o el hormigonado in situ, Hess (26).

En el ámbito nacional, el IRSST y la Escuela de Ingenieros de Caminos, Canales y Puertos de la Universidad Politécnica de Madrid (27) han desarrollado estudios que incluyen el análisis de propuestas técnicas que mejoran las condiciones de trabajo en actividades tales como la señalización y balizamiento de trabajos de conservación de carreteras, la ejecución de estructuras de obra civil, solados o el montaje de vía en obras ferroviarias (Figura 4).

\subsection{Desarrollo internacional de la PtD en los últimos años.}

El desarrollo de las técnicas de PtD en el ámbito internacional ha tomado en los últimos años un impulso notorio que se refleja en los siguientes hechos:

- La OSHA ha formado un grupo de trabajo de diseño para la seguridad en la construcción compuesto por diez organizaciones de profesionales desde 2005.

- El Instituto Nacional de Seguridad y Salud Ocupacional de los EE.UU (NIOSH) ha reconocido la Prevención a través del Diseño como un enfoque de seguridad muy prometedor. En 2006, ésta se convirtió en una de las diez esferas prioritarias del Programa Nacional de Investigación Ocupacional (NORA) del Consejo de Sector de la Construcción. En 2007, NIOSH convocó a un taller de Prevención a través del Diseño con cerca de 300 participantes de ocho sectores de la industria.

- En Australia, la Estrategia Nacional de Seguridad y Salud en el Trabajo, 20022012 incluye la eliminación de riesgos en la fase de diseño como un área de prioridad nacional identificando, como objetivos a lograr tanto construir aplicando dichos conceptos como la impartición de la formación necesaria para lograr tal fin.

- La Asociación de Ingenieros de Seguridad de los EEUU, junto con la Agencia 
Estatal NIOSH han desarrollado un estándar específico para la implantación de las técnicas de PtD. El American National Standard: Prevention Through Design: Guidelines for Addressing Occupational Hazards and Risks in Design and Redesign Processes, ASSE, ANSI/ASSE Z590.32011 vigente desde Enero de 2012, aplica técnicas de PtD y análisis de diseños más seguros en las fases de planificación y proyecto, ejecución, post-incidente y de explotación.

En cuanto a las vías de desarrollo de la PtD, Toole y Gambatese (28) proponen cuatro trayectorias específicas para el futuro de la $\mathrm{PtD}$ en la construcción:

- Potenciar un aumento de la prefabricación que permita la mejora de las condiciones de trabajo en las que se ejecutan buena parte de los trabajos reduciendo, especialmente, los riesgos relacionados con los trabajos en altura y el entorno.

- Aumentar el uso de materiales y sistemas y equipos constructivos menos peligrosos. Especialmente en lo que se refiere a productos tales como materiales para recubrimientos, adhesivos y productos de limpieza y, en general, a las soluciones que permiten la automatización de ciertos trabajos de riesgo.

- Mayor aplicación de ingeniería de la construcción. De manera que la ingeniería amplíe sus objetivos de diseño hacia el campo de la prevención de riesgos laborales analizando soluciones innovadoras tales como el desarrollo de sistemas de retención y sujeción, de estructuras provisionales más seguras, la habilitación de puntos de anclaje para el control del riesgo de caída en altura... Con todo ello, se pretende que el proyectista incorpore el diseño del proceso de construcción en sus procedimientos y actuaciones.

- Consideración desde el diseño de la obra de los espacios de trabajo. De forma que se habiliten puestos de trabajo accesibles y ergonómicos y se consideren, desde esta fase, tanto las distancias de seguridad a observar a los riesgos existentes como las dimensiones necesarias para permitir un acceso y trabajo seguro en las posteriores fases de ejecución, mantenimiento y servicio.

4. APUNTES PARA MEJORAR LA INTEGRACIÓN DE LA PREVENCIÓN EN EL PROYECTO EN ESPAÑA: EL PROMOTOR COMO AGENTE CRÍTICO PARA SU IMPLEMENTACIÓNY DESARROLLO

Si bien España es uno de los países en los que existe una regulación objetiva de la obligación de integrar la prevención en la elaboración del proyecto, no es menos cierto que, en la práctica, el requerimiento que sobre tal objetivo llevan a cabo las autoridades competentes ${ }^{7}$ y los propios promotores de obras de construcción resulta escaso cuando no inexistente. Dicha situación se ve confirmada por el hecho habitual de que los promotores y proyectistas limiten, en la práctica, su gestión preventiva en esta fase a la mera elaboración del Estudio de Seguridad y Salud. De esta manera, si bien formalmente se da cumplimiento a una parte de los deberes preventivos vigentes, se termina por obviar tanto la preceptiva evitación de los riesgos en su origen como la implementación y desarrollo de soluciones técnicas desde el proyecto que mejoren los niveles de seguridad de los trabajos de construcción, mantenimiento y explotación de la futura obra.

Ante esta situación se entiende que el promotor de la obra es, sin duda, el agente crítico para lograr tanto la utilización de las técnicas de PtD analizadas en los epígrafes precedentes como, en un ámbito más general, la efectiva consideración de la prevención en las etapas previas al comienzo de las obras (que incluyen desde la planificación y elaboración del proyecto hasta la contratación de la misma). Los atributos que justifican la capacidad del promotor de la obra para llevar a cabo este cambio de paradigma son los siguientes:

- En primer lugar, en su faceta de cliente del proceso productivo resulta evidente su influencia en lo que se refiere al establecimiento de las prioridades del mismo. De esta manera, se entiende que en todo proyecto la planificación de costes, plazos, objetivos y soluciones técnicas y constructivas pasa inexorablemente bien por la definición directa por parte del promotor, bien por su aprobación.

- En la misma línea, el mero hecho de que sea el promotor el que seleccione al proyectista que va a diseñar la obra, supone que difícilmente se incluirá la prevención como uno de los objetivos del proyecto si no se requiere su efectiva integración desde los procesos de selección y contratación de dicho agente.

- Adicionalmente, en la mayor parte de los proyectos el promotor es el único agente que permanece a lo largo de todo el ciclo de vida de la obra. Así, no sólo planifica, diseña y contrata la obra sino que, en general, controla y monitoriza su ejecución para, en no pocos casos, gestionar su mantenimiento y explotación una vez concluidos los trabajos de construcción. De esta manera, debería ser el primer interesado en lograr que, desde el diseño de la obra, se habilitasen las soluciones
Resulta Ilamativo que el Texto Refundido de la Ley de Infracciones y Sanciones en el Orden Social, RDL 5/2000, limite la configuración de eventuales infracciones del promotor en fase de proyecto a la ausencia de designación del Coordinador en esta fase y a la inexistencia o insuficiencia del Estudio de Seguridad y Salud cuando el Art. 8 del RD 1627/97 prescribe, además, la obligada consideración de los principios preventivos en el diseño de la obra. 
5. Curvas de eficacia de las diferentes actuaciones preventivas en la fase previa al comienzo de las obras. Base informe Lorent y Puig et al (28). técnicas para mejorar las condiciones de ejecución y explotación de la obra que promueve al estar, ambas, íntimamente relacionadas con las decisiones tomadas en fase de diseño.

- Por último, no se deben obviar las ventajas que la aplicación de las técnicas de PtD suponen para la definición del proyecto y la mejora que éstas suponen en lo referente a la planificación global de los trabajos lo que, en la práctica, redundará en los intereses del promotor de la obra.

En base a todo ello, parece claro que las actuaciones preventivas a poner en práctica por el promotor de las obras se deben centrar en la anticipación y, por lo tanto, concentrarse en las etapas previas al comienzo de las obras, cuando la modificación o subsanación de deficiencias no resulta tan costosa en términos económicos y de cumplimiento de plazos y condiciones de ejecución (Figura 5).

Considerando el potencial que guardan las técnicas de $\mathrm{PtD}$, no parece lógico que el proyectista limite su aportación preventiva al proceso a la concepción de la obra, la definición del proyecto y, posteriormente, a la comprobación de que la obra ejecutada se ajusta al diseño y a las especificaciones previstas. Si bien dichas actuaciones son necesarias y concentran buena parte las aportaciones de los proyectistas, no resulta desdeñable añadir a las mismas dos esenciales en lo que respecta al éxito preventivo del proceso: en primer lugar la obligación de considerar como aspecto esencial de todo proyecto su viabilidad constructiva y, de la mano de la primera, su viabilidad preventiva. Todo ello, implica la necesaria integración de los aspectos preventivos a la hora de definir las futuras soluciones constructivas para las fases de ejecución y explotación y mantenimiento.

Y todo ello sin obviar que la organización de la obra, en lo que se refiere a la disposición concreta de sus tajos, empresas y trabajadores participantes competerá al empresario contratista el cual, en todo
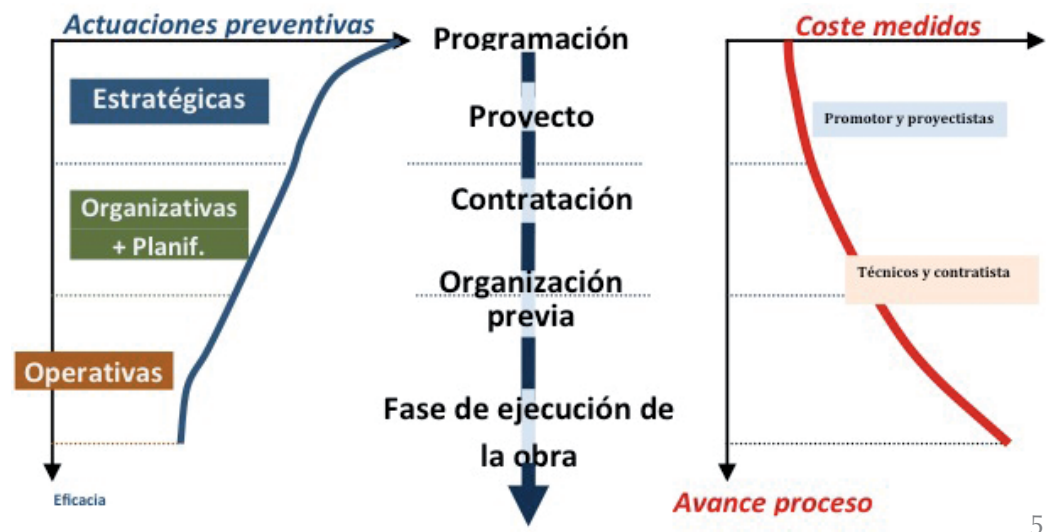

caso, se verá muy favorecido en términos preventivos por una información adecuada y, sobre todo, por los avances preventivos logrados en esta etapa inicial en relación con los riegos evitados o minorados en el propio proyecto de construcción.

De es esta manera, se pretende que la aportación del promotor de la obra en esta fase no se limite a la necesaria consideración y resolución de los aspectos relacionados con la funcionalidad, durabilidad, economía y estética de la futura obra, sino que se incluyan en los criterios de decisión y definición técnica de la obra los relacionados con la gestión preventiva del edifico, instalación o infraestructura en sus diferentes etapas.

\section{CONCLUSIONES Y FUTURAS VÍAS DE DESARROLLO E INVESTIGACIÓN}

Tras el análisis realizado y en base a las referencias bibliográficas citadas, las conclusiones más relevantes en relación con la integración de la prevención en la fase de proyecto y la utilización de la técnicas de PtD son las siguientes:

1. Un porcentaje ciertamente relevante de los accidentes laborales acaecidos en las obras de construcción (entre el 35\% y el $50 \%$ según el estudio e investigador de referencia), guarda relación con las decisiones técnicas tomadas en el diseño de la obra en fase de proyecto. Ello no significa que la causa exclusiva de los mismos sean las deficiencias preventivas de los proyectos si bien resulta claro que un avance, en términos preventivos, de los mismos sería decisivo para la mejora de las condiciones de trabajo en las fases de ejecución y mantenimiento y explotación de los edificios e infraestructuras proyectadas.

2. Es preciso revisar y modificar los actuales modelos de análisis causal de los accidentes laborales en el sector. Para ello, es preciso trascender del mero estudio de las causas directas que los originan para analizar la contribución que, desde el proyecto, se puede realizar para evitarlos. Mediante tal análisis, no se pretende culpabilizar al proyectista (pues en todo caso, la especificación o solución adaptada en proyecto habría de ser implementada y atendida por los empresarios de la obra), sino facilitar la evitación de accidentes repetitivos y procurar la mejora continua desde la propia definición técnica de los trabajos en fase de proyecto.

3. La Directiva Comunitaria 92/57/CEE obliga a la propiedad (promotor) y los proyectistas a integrar los aspectos preventivos en 
el diseño de la obra. Ello significa, entre otros objetivos, que en las decisiones técnicas que definen el diseño de la futura obra de construcción se deberá priorizar la evitación de los riesgos laborales combatiendo éstos desde el origen del proceso productivo -origen que, en la construcción, se localiza en el diseño y planificación de la obra que se realiza en la fase de proyecto. Igualmente, el RD 1627/97 obliga a los proyectistas a integrar la prevención en la elaboración del proyecto considerando los principios aludidos.

4. La integración de la prevención en el diseño de la obra en el ámbito comunitario dista de ser efectiva y exige de un mayor desarrollo e implicación de promotores y proyectistas. En España, las modificaciones operadas en la regulación de la gestión preventiva de la fase de proyecto sobre lo previsto en la Directiva 92/57/ CEE (dualidad documental y vinculación de la obligación de designar al coordinador de seguridad en fase de proyecto a la participación de más de un proyectista), dificultan la debida integración de la prevención en esta fase.

5. Las técnicas de Prevención a través del Diseño (PtD), que incluyen el análisis y desarrollo desde el proyecto de soluciones más seguras como pueden ser el aumento de la prefabricación, la utilización de equipos y materiales más seguros o la automatización de procesos, se configuran como herramientas de trabajo de elevado potencial no sólo para cumplir los requisitos legales vigentes, sino para lograr una mejora efectiva de la prevención desde el diseño de la obra. Las ventajas que acarrea una correcta integración de la prevención en el proyecto residen en la anticipación en la gestión de los riesgos, la limitación de la improvisación o el aprovechamiento de la innovación tecnológica y permiten desplazar el concepto de la seguridad en la construcción desde la ámbito de la protección hacia los de la ingeniería y la prevención.

6. La adopción de dichas técnicas no está dirigida a eximir de responsabilidad a las empresas que ejecutan los trabajos pero sí trata de cambiar una cultura, la actual, más basada en buscar culpables que en compartir la responsabilidad que el global de los agentes ostentan para mejorar las condiciones de trabajo existentes a lo largo de todo el ciclo de vida de una obra de construcción.

7. La integración de la prevención en esta fase y la puesta en práctica de las tecnologías automatizadas puede verse facilitada si, durante el proceso de diseño, se ofrecen al contratista oportunidades para incorporarlas. Todo ello aconseja que, desde la fase de proyecto, se promueva una colaboración fluida entre el constructor y el diseñador lo que, al menos en el espectro español, se ve dificultado por los sistemas de contratación más habitualmente utilizados en el sector.

8. La integración de la prevención en el proyecto y la aplicación de las técnicas de PtD están directamente relacionadas con aspectos tales como la sostenibilidad en la construcción (pues su enfoque incluye todo el ciclo de vida de la obra) o la economía del diseño (pues priorizan el diseño de soluciones técnicas desde la ingeniería frente a soluciones costosas a improvisar en fase de ejecución).

En cuanto a los retos pendientes y las vías de investigación a desarrollar en esta materia se apuntan los siguientes:

- Se considera necesario desarrollar una metodología que facilite a los organismos públicos con competencias en la materia el análisis técnico de los accidentes laborales en la construcción. Con ello se lograría ir más allá del mero estudio de causas directas que los motivan que, en realidad, poco o nada aportan en términos de facilitar la evitación de la repetición de los mismos. En esta línea, se considera necesario analizar e implementar modelos de investigación de accidentes que valoren la eventual contribución causal de las decisiones técnicas tomadas en fase de proyecto.

- En lo que se refiere a la innovación en prevención en la construcción, se considera necesario concretar vías específicas de desarrollo (recomendaciones, guías de buenas prácticas) y, además, que se habiliten los cauces y medios necesarios para su difusión entre los agentes del sector.

- En relación con la necesaria participación de los proyectistas en la integración de la prevención en el diseño de la obra, se considera útil promover el análisis y desarrollo de herramientas y metodologías destinadas a facilitar tal labor.

- Por último, en lo que se refiere a los aspectos formativos, y al igual que ya sucede en países tales como Irlanda, Alemania, Bélgica o Francia, se considera especialmente crítico que se integren los contenidos preventivos de manera efectiva en los programas formativos de las ingenierías y carreras técnicas que habilitan para la elaboración de proyectos de construcción facilitando, así, que tal actuación profesional termine por integrar las consideraciones preventivas. 


\section{REFERENCIAS}

(1) Martín-Hernández, M. L. (2007). Inefectividad de la normativa de prevención de riesgos laborales y siniestralidad laboral en España: una relación de causa-efecto. Revista de Derecho Social, 40: 180.

(2) Lorent, P. (1991). From drawing board to building site: working conditions, quality and economic performance, p.64. European Foundation for the Improvement of Living and Working Conditions, Dublin (Ireland).

(3) Haslam, R., et al. (2003). Causal factors in construction accidents. Health and Safety Executive Research Report, 156. HMSO Books.

(4) Smallwood, J. (1996). The Influence of Designers on Occupational Safety and Health. Primera Conferencia Internacional de la CIB Working Commission W99. Lisboa, Portugal.

(5) Bennett, L. (2004). Peer review of analysis of specialist group reports on causes of construction accidents. Health and Safety Executive Research Report, 218.

(6) Haslam, R., et al. (2005). Contributing factors in construction accidents. Applied Ergonomics. 36: 401-415.

(7) Behm, M. (2006). Linking Construction Fatalities to the Design for Construction Safety Concept. Safety Science, 43: 589-611.

(8) Behm, M. (2006). An Analysis of Construction Accidents from a Design Perspective. The Center to Protect Workers' Rights.

(9) National Occupational Health and Safety Commission. (2004). The role of design issues in workrelated injuries in Australia. National Occupational Health and Safety Commission, Commonwealth of Australia.

(10) SEOPAN. (2006) Informe sobre la accidentalidad laboral en el sector de la construcción. Sistema Delt@ 2003-2005.p. 200,ANCOP.

(11) Ramos-Pereira, L. D., et al. (2011). Investigación sobre factores relacionados con los accidentes laborales mortales en el sector de la de edificación -trienio 2008-2010. p. 55, Fundación MUSAAT.

(12) Martínez-Aires, M. D., Rubio, M. C., Gibb, A. (2010). Prevention through design: The effect of European Directives on construction workplace accidents. Safety Science, 48(2): 248-258.

(13) Comisión Europea. (2008, 12 de noviembre). Comunicación de la Comisión Europea al Consejo, al Parlamento Europeo, al Comité Económico y Social Europeo y al Comité de las Regiones relativa a la aplicación práctica de las Directivas 92/57/CEE (obras de construcción temporales o móviles) y 92/58/CEE (señalización de seguridad en el trabajo) en materia de salud y seguridad en el trabajo. Bruselas.

(14) European Commission. (2011). Non-binding guide to good practice for understanding and implementing Directive 92/57/CE. Construction Sites, p. 185. Publications Office of the European Union, Luxembourg. 10.2767/31797.

(15) Anduiza, R., Ros, A. (2009). Análisis de los documentos preventivos, su eficacia en la integración de la seguridad y salud en el proceso constructivo y su incidencia en los accidentes laborales. $V$ Convención Técnica y Tecnológica de la Arquitectura Técnica.

(16) ILO, International Labor Office. (1985). Safety and Health in Building and Civil Engineering Work, p.386. International Labor Office.

(17) US Department of Commerce, National Bureau of Standards, Investigation of L'Ambiance Plaza Building Collapse in Bridgeport, Connecticut, NBSIR 87-3640, Ex.34. Gaithersburg, MD September 1987.

(18) Hinze, J., Wiegand, F. (1992). Role of designers in construction worker safety. Journal of Construction Engineering and Management, 118(4): 677-684.

(19) Gambatese, J., Hinze, J., Haas, C. (1997). Tool to Design for Construction Worker Safety. Journal of Architectural Engineering American Society of Civil Engineers (ASCE), 3(1): 32-41.

(20) Szymberski, R. (1997). Construction project safety planning. Tappi Journal, 80(11): 69-71.

(21) Korman, R. (1999). Undeserved attention. Designers say OSHA is unfairly expanding safety responsibility without clear legal basis. Engineering News Record, 21: 28-32.

(22) Gambatese, J., Behm, M., Hinze, J. (2005). Viability of Designing for Construction Worker Safety. Journal of Construction Engineering and Management, 131(9): 1029-1036.

(23) Bendig, M., Gibb, A. (2003). The case for CDM: better safer design-a pilot study, p.76, Health and Safety Executive.

(24) Kramer, D., et al. (2009). Spreading good ideas: A case study of the adoption of an innovation in the construction sector. Applied Ergonomics, 40: 826-832.

(25) Saurin, T., Buarque de Macedo, L., (2008). Ergonomic assessment of suspended scaffolds. International Journal of Industrial Ergonomics, 38: 238-246.

(26) Hess, J., Hecker, S., Weinstein, M., Lunger, M. (2004). A participatory ergonomics intervention to reduce risk factors for low-back disorders in concrete laborers. Applied Ergonomics, 35: 427-441.

(27) Fundación Agustín de Betancourt. (2011). Guía de Buenas Prácticas en prevención de riesgos laborales en obra civil, p. 204, Instituto Regional de Seguridad y Salud en el Trabajo de la Comunidad de Madrid, Madrid.

(28) Toole, T. M., Gambatese, J. (2008). The Trajectories of Construction Prevention through Design. Journal of Safety Research, 39(2): 225-230.

(29) Puig, R., Gili, S. y Montanera, J. M. (2010 ). Análisis de las funciones de coordinación de seguridad y salud en la construcción, p.132, Generalitat de Catalunya, Departament de Treball, Direcció General de Relacions Laborals, Barcelona. 